\title{
Wirksamkeit und Verträglichkeit eines homöopathischen Komplexmittels (Sinusitis Hevert SL) bei akuter, unkomplizierter Rhinosinusitis: Eine multizentrische, randomisierte, doppelblinde, placebokontrollierte Studie an erwachsenen Patienten
}

\author{
Andreas Michalsen ${ }^{a, b}$ Jürgen Palm ${ }^{c}$ Holger Samer $^{d}$ Jan-Christoph Wollmann $^{\mathrm{e}}$ \\ Sieglinde Tausend ${ }^{e}$ Isabel Klein ${ }^{f}$ Jörg Schnitker $^{g}$ \\ a Institute of Social Medicine, Epidemiology and Health Economics, Charité - University Medical Centre, Berlin, Germany; \\ ${ }^{\mathrm{b}}$ Immanuel Hospital Berlin, Department of Internal and Complementary Medicine, Berlin, Germany; ${ }^{\mathrm{c}}$ Dr. med. Jürgen Palm,

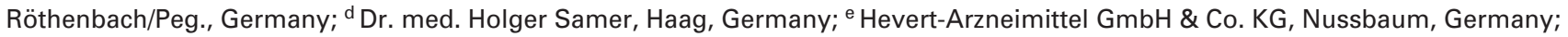 \\ ${ }^{f}$ AtoZ-CRO GmbH, Overath, Germany; ${ }^{9}$ Institut für angewandte Statistik Dr. Jörg Schnitker GmbH, Bielefeld, Germany
}

\section{Schlüsselwörter}

Akute Rhinosinusitis · Komplexmittel · Homöopathie ·

Major Rhinosinusitis Symptom Score (MRSS)

Sino-Nasal Outcome Test 20 (SNOT-20) · Sinusitis Hevert SL

\section{Zusammenfassung}

Hintergrund: In der vorliegenden Studie wurden die Wirksamkeit und Verträglichkeit des homöopathischen Arzneimittels Sinusitis Hevert SL an Patienten mit akuter Rhinosinusitis untersucht. Methoden: In einer randomisierten, placebokontrollierten klinischen Studie wurden 314 Patienten mit bis zu 3 Tagen bestehenden Symptomen, leichtem bis moderatem Gesichtsschmerz und einem Major Rhinosinusitis Symptom Score (MRSS) $\geq 8$ und $\leq 14$ für 15 Tage (Tag 0 erster Arztbesuch plus 14 Tage Behandlung) eingeschlossen und mit Sinusitis Hevert SL oder Placebo behandelt. Den primären Endpunkt bildeten die Responderrate im MRSS (Reduktion $\geq 50 \%$ ) bei der Abschlussvisite sowie die Rate der Remissionen (komplettes Abklingen aller 5 Hauptsymptome). Sekundäre Zielkriterien bildeten die Einschätzung der Wirksamkeit durch den Prüfer (auf einer 4-Punkte-Skala) und der Sino-Nasal Outcome Test 20 German Adapted Version (SNOT-20 GAV; Selbsterhebung durch die Patienten). Ergebnisse: 314 Patienten wurden in die Studie eingeschlossen. Davon wurden 308 mit der Studienmedikation behandelt: 153 mit Sinusitis Hevert SL und 155 mit Placebo. 265 Patienten schlossen die Studie vollständig und valide ab (Valid Completers). Die MRSS $_{\text {pat }}$-Responderrate aller Patienten war für Sinusitis Hevert SL nicht signifikant besser $(85,6 \%$ vs. 80,6\%; $p=0,243$ ), die Rate der Remissionen war mit 31,4\% für Sinusitis Hevert SL und $37,4 \%$ für Placebo in beiden Gruppen nicht signifikant unterschiedlich ( $p=0,2641)$. In der sekundären Completer-Analyse zeigte sich eine signifikante Differenz der Responderraten von $92,1 \%$ versus $83,5 \%$ zugunsten von Sinusitis Hevert SL $(p=0,032)$. Weiter war die Zeit bis zur Verbesserung der Rhinorrhoe signifikant kürzer (4,0 vs. 6,0 Tage; $\mathrm{p}=0,040)$. Vor allem relevante nasale Symptome des SNOT-20 und die Lebensqualität verbesserten sich unter Behandlung mit Sinusitis Hevert SL signifikant deutlicher. Schlussfolgerungen: Es zeigte sich in dieser Studie bei akuter Rhinosinusitis und hoher Placebo-Ansprechrate keine Überlegenheit für Sinusitis Hevert SL in der Gesamtresponder- und Remissionsrate. Es fand sich ein Nutzen von Sinusitis Hevert SL in der Auswertung der Valid Completers für die Response sowie für spezifische Verbesserungen klinisch relevanter Symptome und die Abheilungsgeschwindigkeit der akuten Rhinosinusitis bei guter Verträglichkeit.

(c) 2017 The Author(s). Published by S. Karger GmbH, Freiburg

\section{Keywords}

Acute rhinosinusitis - Complex homeopathic remedy · Homeopathy Major Rhinosinusitis Symptom Score (MRSS) - Sino-Nasal Outcome Test 20 German Adapted Version (SNOT-20) · Sinusitis Hevert SL

\section{Summary}

Efficacy and Safety of a Complex Homeopathic Remedy (Sinusitis Hevert SL) Used in Acute, Uncomplicated Rhinosinusitis: A Multicenter, Randomized, Double-Blind, Placebo-Controlled Trial in Adults

Background: We aimed to assess the efficacy and safety of the complex homoeopathic remedy Sinusitis Hevert SL in patients with acute rhinosinusitis. Methods: In a randomized, placebo-controlled clinical trial, patients with symptoms existing for $\leq 3$ days, with mild to moderate facial pain and a Major Rhinosinusitis Symptom Score (MRSS) of $\geq 8$ and $\leq 14$ were included and treated with either Sinusitis Hevert SL or placebo for 15 days (day 0 first presentation plus 14 days of treatment). Primary endpoints were the responder rate (reduction of $\mathrm{MRSS}_{\text {pat }} \geq 50 \%$ ) at the final visit and the remission rate ( 5 main symptoms vanished). Further outcomes included the assessment of efficacy by the investigator (4point scale) and the Sino-Nasal Outcome Test 20 German Adapted Version (SNOT-20 GAV; patient assessed). Results: Of 314 included patients, 308 were treated: 153 with verum and 155 with placebo. 265 patients completed the trial validly (Valid Completers). The primary outcomes were not significantly different between groups (responder rate MRSS $_{\text {pat }} 85.6 \%$ vs. $80.6 \%$; $=0.243$; remission rate $31.4 \%$ vs. $37.4 \%$; $p=$ 0.264). In the Valid Completers analysis, the $\mathrm{MRSS}_{\text {pat }}$ responder rate in the verum group was significantly higher $(92.1 \%$ vs. $83.5 \% ; p=0.032)$ and the time to improvement of the anterior rhinorrhoea was shorter ( 4.0 vs. 6.0 days; $p=0.040$ ). Especially the primary nasal symptoms of the SNOT-20 and the quality of life improved faster under the verum. Conclusions: Sinusitis Hevert SL does not lead to higher responder and remission rates under the condition of high placebo response rates. Data of the Valid Completers analyses indicate a possible faster cure of rhinosinusitis and improvement of some clinically relevant symptoms due to the preparation, which was well tolerated.

\section{KARGER}

Fax +497614520714 Information@Karger.com www.karger.com

๑ 2017 The Author(s)
Published by S. Karger GmbH, Freiburg
Open access
This article is licensed under the Creative Commons AttributionNon Commercial-NoDerivatives 4 .0 International License (CC BY-NCND 4.0) (http://www.karger.com/Services/OpenAccessLicense). Usage (1)
Univ.-Prof. Dr. med. Andreas Michalsen

Zentrum für Naturheilkunde

Immanuel Krankenhaus Berlin

Am kleinen Wannsee 5, 14109 Berlin, Deutschland

a.michalsen@immanuel.de 


\section{Einleitung}

Eine akute Rhinosinusitis (ARS) ist eine gleichzeitige Entzündung der Mucosa von Nase und Nasennebenhöhlen. Sie ist eine der 10 häufigsten Erkrankungen in der Primärmedizin und der fünfthäufigste Anlass für die Verschreibung von Antibiotika [1]. Weltweit hat die ARS eine Prävalenz von 6-15\%, übt einen erheblich negativen Einfluss auf die Lebensqualität aus und verursacht hohe sozioökonomische Kosten [2, 3].

Die überwiegende Mehrzahl der ARS-Fälle ist viralen Ursprungs, während akute bakterielle Infektionen nur in 0,5-2\% der Fälle auftreten [2, 4]. Aufgrund der Einschränkung der Lebensqualität und der hohen sozioökonomischen Kosten werden therapeutisch eine Heilung der akuten Symptome, eine Verkürzung der Genesungszeit sowie - im Falle einer medikamentösen Therapie idealerweise die Abwesenheit von unerwünschten Arzneimittelwirkungen angestrebt.

Die konventionelle Standardtherapie der ARS ist in 2 (derzeit nicht aktuellen) Leitlinien festgehalten $[1,5]$. Sie zielt auf eine Milderung der Symptome sowie auf eine Verkürzung der Krankheitsdauer ab. Eingesetzt werden können der Leitlinie der Deutschen Gesellschaft für Allgemein- und Familienmedizin (DEGAM) zufolge Analgetika bzw. Antiphlogistika, Dekongestiva und gegebenenfalls topische bzw. systemische Kortikoide. Für «sonstige pharmakologische Therapien» wie Sekretolytika oder Phytotherapeutika werden keine bzw. heterogene Empfehlungen gegeben [1]. In der Aufstellung der Leitlinie der Deutschen Gesellschaft für HalsNasen-Ohren (HNO)-Heilkunde, Kopf- und Halschirurgie, können lokal oder systemisch verabreichte Sympathomimetika oder Parasympatholytika, lokal angewandte salzhaltige Lösungen (Sprays oder Inhalationen), Sekretolytika, Analgetika bzw. Antiphlogistika und andere Pharmaka verabreicht werden [5]. Beiden Leitlinien zufolge sollen Antibiotika nur bei schweren Verlaufsformen und besonderen Patientengruppen eingesetzt werden. Auch wird zunehmend wahrgenommen, dass Antibiotika bei akuter oder chronischer - selbst bei bakteriell induzierter - Rhinosinusitis nur eine sehr limitierte Wirksamkeit zeigen [1, 5-10]. Außerdem weisen Daten der letzten Jahre auf eine zunehmende Bildung von Antibiotikaresistenzen, vermutlich auch aufgrund wenig gezielter Antibiotikatherapien [11].

Gerade auch aus Gründen der erwähnten Resistenzbildung erfahren komplementärmedizinische Behandlungen zunehmende Aufmerksamkeit, wobei bevorzugt auch pflanzliche oder homöopathische Arzneimittel eingesetzt werden. Erhebungen nach nutzen 15-20\% der Patienten mit ARS homöopathische oder pflanzliche Medikamente [2, 12-14]. Vor allem für homöopathische Arzneimittel ist jedoch die Anzahl qualitativ hochwertiger klinischer Studien (doppelblind, placebokontrolliert, randomisiert) sehr begrenzt. Bislang gibt es nur 1 Studie, die ebenfalls die Behandlung von ARS untersucht [15].

Sinusitis Hevert SL ist ein homöopathisches Arzneimittel in Tablettenform, das zur Behandlung von Entzündungen des HalsNasen-Rachenraumes und der Nasennebenhöhlen (Sinusitis) zugelassen ist [16]. Als sogenanntes «Komplexmittel» besteht es aus
Tab. 1. Zusammensetzung von Sinusitis Hevert SL

\begin{tabular}{llc}
\hline Wirkstoff & Potenz & Menge, mg \\
\hline Apis & D4 & 10 \\
Baptisia & D4 & 5 \\
Cinnabaris & D3 & 5 \\
Echinacea & D2 & 30 \\
Hepar sulfuris & D3 & 10 \\
Kalium bichromicum & D8 & 30 \\
Lachesis & D8 & 10 \\
Luffa & D4 & 60 \\
Mercurius bijodatus & D9 & 70 \\
Silicea & D2 & 5 \\
Spongia & D6 & 10 \\
\hline
\end{tabular}

Sonstige Bestandteile: Laktosemonohydrat, Magnesiumstearat, Maisstärke.

11 homöopathischen, niedrig potenzierten Einzelmitteln, die sich in ihren Wirkspektren ergänzen (Tab. 1). Die Wahl der Wirkstoffe verfolgt das Ziel, nicht nur die Symptome der Entzündungen des Hals-Nasen-Rachenraumes oder der Nasennebenhöhlen zu bekämpfen, sondern auch die körpereigenen Abwehrkräfte zu stärken. Sinusitis Hevert SL ist seit 2007 in der aktuell bestehenden Kombination als Arzneimittel durch das Bundesamt für Arzneimittel und Medizinprodukte (BfArM) zugelassen und kann in Apotheken rezeptfrei erworben werden. Die Wirksamkeit und Unbedenklichkeit von Sinusitis Hevert SL sind bislang durch die im Rahmen der Zulassung vorgelegten Unterlagen (vor allem Monografien der Kommission D beim BfArM) belegt. Da doppelblinde, placebokontrollierte und randomisierte klinische Studien als «Goldstandard» in der klinischen Forschung gelten, wurde diese Studie durchgeführt, um die Wirksamkeit dieses häufig angewandten homöopathischen Arzneimittels weiter zu evaluieren. Ziel war es, die Wirksamkeit, Sicherheit und Verträglichkeit von Sinusitis Hevert SL über eine Behandlungsperiode von 14 Tagen aufzuzeigen.

\section{Patienten und Methoden}

Die randomisierte, doppelblinde, placebokontrollierte Phase-IV-Parallelgruppenstudie wurde von November 2014 bis April 2015 an 23 Zentren (6 HNO-Fachärzte, 9 Allgemeinmediziner und 4 auf die Durchführung von Studien spezialisierte Zentren, 3 Therapiezentren, 1 Klinik Abteilung Naturheilkunde) in Deutschland durchgeführt. Die Studie folgte den Standards der Deklaration von Helsinki, der International Council for Harmonisation (ICH) Harmonised Tripartite Guideline for Good Clinical Practice (CPMP/ ICH/135/95) sowie den in Deutschland gültigen gesetzlichen Vorgaben. Die Ethikkommission des Landesamtes für Gesundheit, Berlin, und das BfArM als zuständige Bundesoberbehörde genehmigten die Studie vor Einschluss des ersten Patienten. Nach Aufklärung der Patienten unterschrieben diese vor dem Beginn studienspezifischer Maßnahmen die Einverständniserklärung.

\section{Einschlusskriterien}

Männliche und weibliche ambulante Patienten zwischen 18 und 75 Jahren mit der klinischen Diagnose einer akuten unkomplizierten oder einer akuten rezidivierenden Rhinosinusitis wurden in die Studie eingeschlossen. Die Symptome durften bei Einschluss nicht länger als $72 \mathrm{~h}$ bestehen, und die Patienten 
mussten einen ärztlich ermittelten Major Rhinosinusitis Symptom Score (MRSS) zwischen 8 und 14 Punkten aufweisen. Der MRSS (Maximum 15 Punkte) wird aus der Summe der 5 Rhinosinusitis-Hauptsymptome gebildet: anteriore und postnasale Rhinorrhoe, nasale Verstopfung, Kopfschmerz und Gesichtsschmerz/-druck (beim Bücken) [17]. Jedes Hauptsymptom kann mit $0=$ kein/nicht vorhanden, $1=$ leicht, 2 = moderat oder $3=$ stark bewertet werden. Für den Einschluss eines Patienten in die Studie mussten die Symptome nasale Verstopfung, leichter bis moderater Gesichtsschmerz/-druck (beim Bücken) und ein drittes MRSS-Hauptsymptom vorhanden sein. Frauen im gebärfähigen Alter mussten bereit sein, kontrazeptive Methoden anzuwenden.

\section{Ausschlusskriterien}

Ausschlusskriterien waren eine Behandlung mit Immunsuppressiva bis 8 Wochen vor Studieneinschluss, systemisch oder nasal angewandte Antibiotika oder Kortikosteroide innerhalb der letzten 4 Wochen, Erkältungs- oder immunmodulatorische Medikamente während der letzten 7 Tage sowie die Einnahme von alpha-Sympathomimetika innerhalb der letzten $5 \mathrm{~h}$ vor dem Screening. Weitere Ausschlusskriterien bildeten Schwangerschaft oder Stillzeit, Rauchen in den letzten 2 Jahren, eine klinisch relevante Immundefizienz, manifeste Autoimmunerkrankungen, schwere sonstige Grunderkrankungen sowie schwere psychiatrische Erkrankungen.

\section{Prüfmedikation und Begleittherapien}

Als Behandlungsdauer wurden 15 Tage gewählt (Tag 0 als Tag der Erstkonsultation, Visite 1, plus 14 Tage). Die Studienmedikation wurde entsprechend der zugelassenen Dosierungsempfehlung biphasisch eingenommen: in der 1. Woche in einer Dosierung von $6 \times 2$ Tabletten/Tag, in der 2 . Woche in einer Dosierung von $4 \times 2$ Tabletten/Tag. Die Einnahme erfolgte in vorgegebenen Zeitfenstern. Am Tag der ersten Konsultation (Tag 0, Visite 1) erhielten die Patienten nur die Anzahl an Tabletten, die durch diese vorgegebenen Zeitfenster für den Tag verblieben waren. Als Notfallbehandlung bei unzureichender Wirkung waren Inhalation mit Wasserdampf und die Einnahme von Paracetamol erlaubt. Soweit mit den Ausschlusskriterien vereinbar war die Behandlung von Begleiterkrankungen uneingeschränkt zulässig.

\section{Parameter der Wirksamkeit}

Ärztliche Erhebungen fanden am Tag 0 (Erstkonsultation, Visite 1) und an den Tagen 7, 10 und 14 (Visiten 2, 3, 4) statt. Neben der Ersterhebung des ärztlichen MRSS $_{\text {inv }}($ inv = Investigator, Prüfarzt) an Tag 0 erfolgten Kontrollen

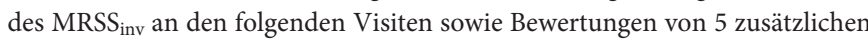
mit Rhinosinusitis/Atemwegsinfekt assoziierten Symptomen bei jeder Visite: Halsschmerzen/Hals-, Rachenentzündung; Räusperzwang; Niesen; Husten/ Hustenreiz; Schmerzen/Druckgefühl auf den Ohren. An den Visiten 1 und 4 beurteilten die Prüfärzte den Gesundheitszustand der Patienten mittels einer visuellen Analogskala (Visual Analog Scale, VAS; $10 \mathrm{~cm}$; 0 = bester Gesundheitszustand, 10 = schlechtester Gesundheitszustand) sowie an Visite 4 die generelle Effektivität der Therapie mittels der Skala 1 = Symptome geheilt, 2 = Symptome gebessert, 3 = Symptome unverändert, 4 = Symptome verschlechtert.

Die Patienten füllten bei jeder Visite den Sino-Nasal Outcome Test $20 \mathrm{Ger}$ man Adapted Version (SNOT-20 GAV) aus. Dieser Test ist ein validiertes Instrument zur Messung der Lebensqualität bei Rhinosinusitis [18, 19]. Zusätzlich beurteilten die Studienteilnehmer ihren Gesundheitszustand und die Arzneimittelverträglichkeit mittels einer VAS. Die Patienten erhielten Tagebücher, um täglich sowohl den MRSS $_{\text {pat }}$ (pat = Patient) als auch die Einnahme der Studien- und Notfallbehandlung bzw. Nebenwirkungen zu dokumentieren.

\section{Parameter der Verträglichkeit und Sicherheit}

Unerwünschte Ereignisse wurden im Tagebuch registriert und bei jeder Visite in standardisierter Form dokumentiert. Vitalparameter wurden bei jeder Visite gemessen, eine körperliche Untersuchung wurde bei den Visiten 1 und 4 durchgeführt. Die Studienteilnehmer beurteilten die Arzneimittelverträglichkeit an den Visiten 2, 3, und 4 mittels einer VAS $(10 \mathrm{~cm} ; 0=$ beste Verträglichkeit, 10 = schlechteste Verträglichkeit), die Prüfärzte nur an Visite 4.

\section{Adaptives Design und Fallzahlschätzung}

Der erste primäre Endpunkt der klinischen Studie war die Rate der Responder zwischen Visite 1 und der Abschlussvisite (Visite 4). Die Daten hierzu erfasste der Patient täglich durch Beantwortung der Fragen im MRSS ${ }_{\text {pat }}$. Ein positives Ansprechen wurde definiert als stabile Reduktion des MRSS $_{\text {pat }}$ um mindestens 50\% vom Ausgangswert ohne nachfolgende erneute Verschlechterung. Der zweite primäre Endpunkt war die Rate der Remission zwischen Visite 1 und der Abschlussvisite, definiert als komplettes Abklingen aller 5 Hauptsymptome der Rhinosinusitis ohne anschließendem Wiederauftreten auch nur eines Symptoms bis zum Behandlungsende.

Beide Endpunkte wurden als co-primär ausgewertet, d.h. auf Erfolg konnte nur dann konfirmatorisch geschlossen werden, wenn beide Ziele, Response und Remission, erreicht wurden. Alle weiteren Parameter galten als sekundär.

Die Überlegenheit von Sinusitis Hevert SL im Vergleich zu Placebo in Bezug auf die Responderrate wurde in einem 2-stufigen adaptiven Design nach Bauer und Köhne [20] mit einem 1-seitigen Gesamtsignifikanzniveau von $\alpha=$ 0,025 getestet. In diesem Design werden auf einer ersten Stufe des statistischen Vergleichs 2 Niveaus $\alpha_{1}<0,025<\alpha_{0}$ festgelegt und folgende Entscheidungen getroffen:

- vorzeitige Beendigung der Studie wegen Erfolgs, wenn die p-Werte des Vergleichs Verum versus Placebo sowohl für die Responder- als auch für die Remissionsrate die Bedingung $\mathrm{p}<\alpha_{1}$ erfüllten;

- vorzeitige Beendigung der Studie wegen Misserfolgs, wenn wenigstens ein p-Wert die Bedingung $\mathrm{p} \geq \alpha_{0}$ erfüllt;

- Fortsetzung der Studie auf der zweiten Stufe mit dem neuen Niveau $\alpha_{2}=$ $0,0038 / p$, wenn wenigstens ein $p$-Wert die Bedingung $\alpha_{1} \leq p<\alpha_{0}$ und der andere nicht $\mathrm{p} \geq \alpha_{0}$ erfüllt.

Die erste Stufe wurde mit $\alpha_{1}=0,0102$ und $\alpha_{0}=0,5$ durchgeführt. Das Niveau $\alpha_{1}$ wurde als Zielniveau der ersten Stufe verwendet und gleichzeitig die statistisch ungünstigste Lage der angenommenen Therapiedifferenz von $20 \%$ (nämlich $40 \%$ vs. 60\%) für die Fallzahlschätzung gewählt. Dies führte zu einem Bedarf von 145 Patienten pro Gruppe ohne Dropouts. Insgesamt wurden 315 Patienten in die Studie eingeschlossen. Die zweite Stufe wurde nicht begonnen.

\section{Statistische Auswertung}

Für den Vergleich der Responder- und Remissionsraten wurden 2 1-seitige $\chi^{2}$-Tests eingesetzt. Die Tests der weiteren Endpunkte wurden mit einem 2-seitigen Signifikanzniveau von $\alpha=0,05$ durchgeführt. Zur Anwendung kamen folgende statistische Methoden:

- $\quad \chi^{2}$-Test zur Überprüfung von Häufigkeitsverteilungen;

- Logrank-Test zum Vergleich der Kaplan-Meier-Darstellungen von Timeto-Event-Daten;

- Mann-Whitney-U-Test, t-Test zum Vergleich der Behandlungsgruppen unter Berücksichtigung der stetigen Daten (nichtparametrische oder normal verteilte Fälle);

- Wilcoxon-Test für gruppeninterne Vergleiche;

- logistische Regressionsanalyse mit Rückwärtselimination auf dem 15\%-Niveau;

- MMRM-Analyse der Kovarianz für wiederholte Messungen in gemischten Modellen (MMRM = Mixed Model of Repeated Measures) (Visite 2, 3 und 4 vs. Visite 1$)$.

Initial fehlende Angaben der Skala MRSS ${ }_{\text {pat }}$ wurden gemäß Next Observation Carried Backward ergänzt. Für weitere fehlende Daten wurde eine Fortschreibung gemäß Last Observation Carried Forward bis zum letzten Tag der Behandlung vorgenommen.

Die Zuteilung der Patienten in die beiden Behandlungsgruppen, Verum oder Placebo, erfolgte im Verhältnis 1:1 innerhalb von Viererblöcken. Die Randomisierungsliste wurde mit der Funktion RANUNI generiert (SAS ${ }^{\circledR} 9.3$ Functions and CALL Routines 2011; SAS Institute Inc., Cary, NC, USA). Die Übergabe der zugeordneten Prüfmedikation erfolgte demgemäß durch den Prüfarzt.

\section{Verblindung}

Sinusitis Hevert SL und Placebo waren identisch in Aussehen, Konsistenz und Geschmack, sodass weder die CRO (Clinical Research Organisation; 


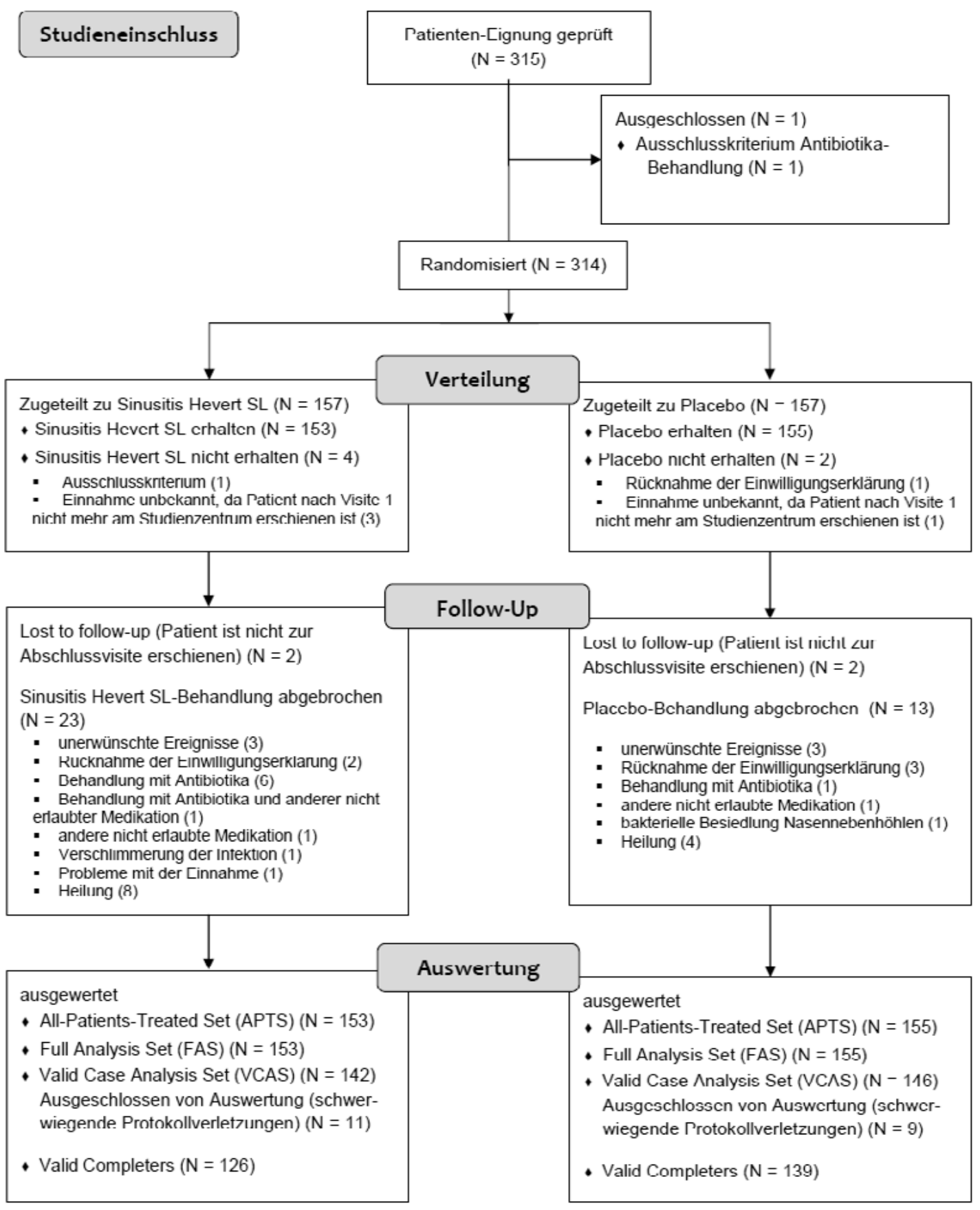

Abb. 1. Übersicht über die Patienten.

Dienstleister für die Durchführung von Studien) noch der Prüfer oder der Patient Kenntnis von der zugeteilten Medikation erlangen konnten. Diese wurde dem Prüfer vor Einsatz eines Medikationsblocks in verschlossenen Notfallkuverts übergeben, deren Unversehrtheit bei Studienabschluss überprüft wurde. Der Therapiecode wurde in keinem Falle vorzeitig gebrochen.

\section{Compliance}

Die Compliance wurde mittels Pill-Counting quantifiziert. Das Pill-Counting wurde für jeden Patienten anhand der verbrauchten Tabletten bzw. der leeren Blisterpackungen durchgeführt. Als compliant galten Patienten mit einer Compliance-Rate $\geq 80 \%$ und $\leq 125 \%$ sowie Patienten mit einer Compliance-Rate $<80 \%$, wenn die niedrige Compliance mit mangelnder Wirksamkeit oder vorzeitiger Heilung begründet wurde.

\section{Ergebnisse}

\section{Kollektive der Auswertung}

Insgesamt wurden in 23 Studienzentren 315 Patienten ausgewählt. Patienten, die das Auswahlverfahren nicht bestanden (Screening Failures, $\mathrm{n}=1$ ), wurden nicht randomisiert. 314 Patienten wurden in die Studie eingeschlossen und einer der beiden Gruppen, Verum oder Placebo, zugeordnet (Intention to Treat (ITT): $\mathrm{n}=314$ ). Sechs Patienten wurden nicht behandelt, das «Full Analysis Set» (FAS) der behandelten Patienten umfasste also 308 Patienten (Sinusitis Hevert SL: $\mathrm{n}=153$; Placebo: $\mathrm{n}=155$; Abb. 1), die uneingeschränkt in die primäre Wirksamkeitsanalyse eingin- 
Tab. 2. Demografische Daten und BaselineCharakteristika im FAS

\begin{tabular}{|c|c|c|c|}
\hline & $\begin{array}{l}\text { Sinusitis } \\
\text { Hevert SL }\end{array}$ & Placebo & p-Wert \\
\hline Anzahl der Patienten, $\mathrm{N}$ & 153 & 155 & \\
\hline Alter, Jahre (Mittelwert \pm SD) & $39,0 \pm 14,9$ & $40,5 \pm 14,5$ & 0,3763 \\
\hline Geschlecht & & & 0,8382 \\
\hline Männlich & $56(36,6 \%)$ & $55(35,5 \%)$ & \\
\hline Weiblich & $97(63,4 \%)$ & $100(64,5 \%)$ & \\
\hline BMI, $\mathrm{kg} / \mathrm{m}^{2}$ (Mittelwert $\left.\pm \mathrm{SD}\right)$ & $26,4 \pm 6,0$ & $26,3 \pm 5,4$ & 0,8577 \\
\hline Typ ARS & & & 0,3396 \\
\hline Unkompliziert & $141(92,2 \%)$ & $147(94,8 \%)$ & \\
\hline Rezidivierend & $12(7,8 \%)$ & $8(5,2 \%)$ & \\
\hline Relevante Vorerkrankungen & & & 0,8418 \\
\hline Nein & $122(79,7 \%)$ & $125(80,6 \%)$ & \\
\hline $\mathrm{Ja}$ & $31(20,3 \%)$ & $30(19,4 \%)$ & \\
\hline Relevante Begleiterkrankungen & & & 0,7276 \\
\hline Nein & $71(46,4 \%)$ & $75(48,4 \%)$ & \\
\hline $\mathrm{Ja}$ & $82(53,6 \%)$ & $80(51,6 \%)$ & \\
\hline MRSS $_{\text {pat }}-$ Hauptsymptome (Mittelwert \pm SD) & $9,37 \pm 2,24$ & $9,26 \pm 2,06$ & 0,6706 \\
\hline MRSS $_{\text {pat }}{ }^{- \text {Nebensymptome }}$ (Mittelwert \pm SD) & $6,54 \pm 2,76$ & $6,35 \pm 2,89$ & 0,5583 \\
\hline SNOT-20 GAV Gesamtscore (Mittelwert \pm SD) & $40,41 \pm 13,50$ & $39,23 \pm 13,63$ & 0,4486 \\
\hline VAS $_{\text {pat }}($ Mittelwert $\pm S D)$ & $6,78 \pm 1,42$ & $6,55 \pm 1,52$ & 0,1736 \\
\hline
\end{tabular}

gen. Patienten, die die Studie wegen Genesung oder aufgrund mangelnder Wirksamkeit vorzeitig beendet hatten, wurden in der primären Wirksamkeitsanalyse berücksichtigt, andere vorzeitige Studienabbrüche $(\mathrm{n}=17)$ oder relevante Protokollverletzungen $(\mathrm{n}=3)$ vom «Valid Case Analysis Set» (VCAS) ausgeschlossen $(\mathrm{n}=288)$. Innerhalb des VCAS wurden $\mathrm{n}=265$ Patienten als Valid Completers analysiert (Abb. 1).

\section{Baseline-Charakteristika}

Die Baseline-Charakteristika wurden im FAS auf Vergleichbarkeit der Prüfgruppen getestet (Tab. 2). Es zeigte sich eine gute Vergleichbarkeit, wenn man von vereinzelten Verteilungsungleichgewichten absieht. So litten Patienten in der Verum-Gruppe fast 3-mal häufiger an Hypothyreose ( $p=0,0486 ; 8,5 \%$ vs. $3,2 \%)$ und zeigten eine stärkere Ausprägung der anterioren Rhinorrhoe ( $p=0,0338$; Anteil stark: $31,4 \%$ vs. $20,9 \%$ ).

\section{Behandlungen}

Die Compliance in der Studie war in beiden Gruppen vergleichbar und war bei insgesamt 92,2\% aller Patienten sehr gut. In der Placebo-Gruppe nutzten geringfügig mehr Patienten Paracetamol als Notfallmedikation (36,6\% vs. 30,7\%; p = 0,2763). Die VerumGruppe wendete nichtsignifikant häufiger Wasserdampfinhalationen an $(21,6 \%$ vs. $18,3 \%$; $\mathrm{p}=0,4743)$.

\section{Explorative Statistik zur Wirksamkeit von Sinusitis Hevert SL (MRSS)}

Eine stabile Abnahme des MRSS $_{\text {pat }}$ um wenigstens 50\% wurde unter Sinusitis Hevert SL in $85,6 \%$ des FAS bzw. 87,3\% des VCAS im Median nach 6 Tagen erreicht. Die Besserungsraten der 5 Hauptsymptome lagen gleichermaßen bei etwa $85 \%$ bis über $90 \%$ (Tab. 3). Eine schnelle Antwort (Median 3 Tage) und hohe Remissionsraten von nahezu $80 \%$ fanden sich für Gesichtsschmerz/druck beim Bücken. Symptomfreiheit wurde für die anderen Hauptsymptome in etwa $50-70 \%$ der betroffenen Patienten erreicht, dennoch betrug der Anteil vollständiger Remissionen, d.h. Verschwinden aller Hauptsymptome, nur gut 30\%. Die Remissionsraten lagen unter Placebo in einem etwas höheren, die Responderrate hingegen in einem geringeren Bereich. Im 2-seitigen Test Sinusitis Hevert SL versus Placebo der Summenscores blieben alle $\mathrm{p}$-Werte unauffällig $(\mathrm{p}>0,2)$.

Konfirmatorische Statistik zur Wirksamkeit von Sinusitis Hevert SL versus Placebo (MRSS)

Der MRSS $_{\text {pat }}$, als erstes primäres Zielkriterium, verbesserte sich über den Behandlungszeitraum von 2 Wochen sowohl in der Sinusitis Hevert SL-Gruppe als auch in der Placebo-Gruppe kontinuierlich. Die MRSS $_{\text {pat }}$-Responderrate über alle Patienten (FAS) war für Sinusitis Hevert SL tendenziell besser, verfehlte jedoch das Signifikanzniveau (Sinusitis Hevert SL vs. Placebo: FAS: $85,6 \%$ vs. $80,6 \%$; $\mathrm{p}=0,2438$ und VCAS: $87,3 \%$ vs. $82,2 \% ; \mathrm{p}=0,2262$ bzw. $\mathrm{p}=0,1219$ und $\mathrm{p}=0,1131$ jeweils 1-seitig). Bei Betrachtung der Valid Completers zeigte sich eine signifikant höhere Responderrate von 92,1\% unter der Behandlung mit Sinusitis Hevert SL im Vergleich zu 83,5\% unter Placebo (2-seitig: $p=0,0320 ; 1$-seitig: $p=0,0170$ ).

Als zweites primäres Zielkriterium war die Remissionsrate zwischen den Gruppen zu vergleichen. Hier fand sich ein Wert von $31,4 \%$ mit Verum versus $37,4 \%$ unter Placebo sowie ein p-Wert 
Tab. 3. Ergebnisse der Behandlung mit Sinusitis Hevert SL und Placebo: MRSS $_{\text {pat }}$ und MRSS $_{\text {inv }}$

\begin{tabular}{|c|c|c|c|c|}
\hline & \multicolumn{2}{|c|}{ Sinusitis Hevert SL } & \multicolumn{2}{|l|}{ Placebo } \\
\hline & FAS & VCAS & FAS & VCAS \\
\hline Anzahl der Patienten, $\mathrm{N}$ & 153 & 142 & 155 & 146 \\
\hline \multicolumn{5}{|l|}{ MRSS $_{\text {pat }}$} \\
\hline Response & $85,6 \%^{1}$ & $87,3 \%^{2}$ & $80,6 \%$ & $82,2 \%$ \\
\hline Remission & $31,4 \%^{3}$ & $33,1 \%^{4}$ & $37,4 \%$ & $39,7 \%$ \\
\hline Zeit bis Response (Median) & 6 Tage & 6 Tage & 7 Tage & 7 Tage \\
\hline \multicolumn{5}{|c|}{$\begin{array}{l}\text { MRSS }_{\text {pat: }} \text { Besserung der Hauptsymptome } \\
\text { (\% der betroffenen Patienten, } \\
\text { medianer Zeitpunkt) }\end{array}$} \\
\hline Anteriore Rhinorrhoe & $84,5 \%$ (4 Tage) & $87,0 \%$ (4 Tage) & $75,2 \%$ (6 Tage) & $76,8 \%$ (6 Tage) \\
\hline Postnasale Rhinorrhoe & $86,3 \%$ (5 Tage) & $86,9 \%$ (5 Tage) & $81,1 \%$ (5 Tage) & $81,9 \%$ (5 Tage) \\
\hline Nasale Verstopfung & $88,2 \%$ (5 Tage) & $88,7 \%$ (5 Tage) & $85,3 \%$ (4,5 Tage) & $86,7 \%$ (4 Tage) \\
\hline Kopfschmerz (beim Bücken) & $85,8 \%$ (5 Tage) & $87,8 \%$ (5 Tage) & $94,3 \%$ (5 Tage) & $95,5 \%$ (5 Tage) \\
\hline $\begin{array}{l}\text { Gesichtsschmerz/-druck } \\
\text { (beim Bücken) }\end{array}$ & $91,5 \%$ (3 Tage) & $92,5 \%$ (3 Tage) & $96,5 \%$ (2 Tage) & $97,0 \%$ (2 Tage) \\
\hline \multicolumn{5}{|l|}{$\begin{array}{l}\text { MRSS }_{\text {inv }} \text { : Remission/Besserung der } \\
\text { Hauptsymptome } \\
\text { (\% der betroffenen Patienten) }\end{array}$} \\
\hline Anteriore Rhinorrhoe & $52,9 \% / 83,7 \%$ & $55,6 \% / 86,6 \%$ & $52,7 \% / 78,7 \%$ & $53,9 \% / 80,9 \%$ \\
\hline Postnasale Rhinorrhoe & $67,4 \% / 83,3 \%$ & $70,7 \% / 85,7 \%$ & $65,7 \% / 83,2 \%$ & $67,9 \% / 85,8 \%$ \\
\hline Nasale Verstopfung & $57,5 \% / 89,5 \%$ & $59,9 \% / 92,3 \%$ & $63,2 \% / 87,1 \%$ & $65,8 \% / 89,7 \%$ \\
\hline Kopfschmerz (beim Bücken) & $69,9 \% / 83,6 \%$ & $73,3 \% / 87,4 \%$ & $78,1 \% / 90,7 \%$ & $81,0 \% / 93,0 \%$ \\
\hline $\begin{array}{l}\text { Gesichtsschmerz/-druck } \\
\text { (beim Bücken) }\end{array}$ & $77,1 \% / 90,2 \%$ & $79,6 \% / 93,0 \%$ & $84,5 \% / 92,9 \%$ & $87,7 \% / 96,6 \%$ \\
\hline
\end{tabular}

2-seitige p-Werte versus Placebo: ${ }^{1} \mathrm{p}=0,2438,{ }^{2} \mathrm{p}=0,2262,{ }^{3} \mathrm{p}=0,2641,{ }^{4} \mathrm{p}=0,2427$.

MRSS $_{\text {pat }}=$ Patientenermittelter Major Rhinosinusitis Symptom Score; MRSS $_{\text {inv }}=$ prüfarztermittelter Major Rhinosinusitis Symptom Score; FAS = Full Analysis Set; VCAS = Valid Case Analysis Set. von 0,8680 (1-seitig). Die Abbruchschranke von $\alpha_{0}=0,5$ wurde damit überschritten, sodass die Studie entsprechend der präspezifizierten Regel für das adaptive Design nach Bauer und Köhne nicht mit einer zweiten Stufe fortgesetzt werden konnte.

\section{Ergänzende Vergleiche zur Wirksamkeit von Sinusitis Hevert SL versus Placebo}

Zur Evaluierung des erhaltenen Ergebnisses wurden Subgruppen- und Verlaufsanalysen durchgeführt. Es wurden verschiedene auffällige Resultate erzielt, unter denen folgende hervorzuheben sind:

- Gesichtsschmerz/-druck (beim Bücken) zeigte unter Verum das vergleichsweise schnellste und stärkste Ansprechen. Allerdings weist gerade dieses Symptom auch den schnellsten und stärksten Placeboeffekt auf, sodass eine signifikante Überlegenheit von Placebo festgestellt werden musste (Responderrate im FAS: 96,5\%; Median der Responsezeit: 2 Tage; $\mathrm{p}=0,0017$ vs. Verum).

- Vorteile von Sinusitis Hevert SL zeigten sich im weiteren Verlauf der Behandlung. So fand sich im FAS eine signifikante Überlegenheit von Verum für die Zeit bis zur Besserung der anterioren Rhinorrhoe (Median 4 Tage unter Verum und 6 Tage unter Placebo; $p=0,0403)$. Die Kovarianzanalyse des Verlaufs von Visite 1 bis Visite 4 lieferte signifikante Zunahmen der Therapiedifferenz Verum minus Placebo für SNOT-
20 Overall Score und SNOT-20 General Quality of Life im VCAS und vor allem für die Gruppe der Valid Completers. Für SNOT-20 Primary Nasal Symptoms blieben gleichsinnige Tendenzen grenzwertig insignifikant, bei Abschluss der Behandlung betrug die Abnahme für die Valid Completers dann jedoch 11,15 \pm 4,47 unter Verum und 9,53 \pm 5,32 unter Placebo mit einer resultierenden Therapiedifferenz von $-1,62$ (95\%-Konfidenzintervall -2,81 bis $-0,42 ; \mathrm{p}=0,0081$ ) zugunsten von Sinusitis Hevert SL.

- Unter den Primary Nasal Symptoms des SNOT-20 GAV zeigte «dickes schleimiges Nasensekret» eine signifikante Überlegenheit von Verum (FAS: $\mathrm{p}=0,0106$; VCAS: $\mathrm{p}=0,0095$ ).

- Das gewählte Kriterium der Remission (Verschwinden aller Hauptsymptome) erwies sich als nicht praxisorientiert, da in beiden Gruppen nur etwa 1 Drittel der Patienten dies erfüllte. Es wurde daher post-hoc eine Analyse mit einem weniger strengen Cut-off von 3 Punkten Restsymptomatik vorgenommen. Hier betrug im Gruppenvergleich die Erfolgsrate für die Valid Completers $86,5 \%$ unter Verum und $77,0 \%$ unter Placebo ( $p=0,0460$ zugunsten von Sinusitis Hevert SL).

- In einer Subgruppenanalyse zeigte sich, dass die Responderrate bei männlichen Patienten in der Sinusitis Hevert SL-Gruppe mit 96,2\% signifikant höher war als in der Placebo-Gruppe mit $83,0 \%(\mathrm{p}=0,0258)$. 


\section{Diskussion}

Die wissenschaftliche Evaluation homöopathischer Fertigarzneimittel durch randomisierte, placebokontrollierte klinische Studien ist bislang nur sehr begrenzt durchgeführt worden. Mit der Durchführung der vorliegenden Studie sollten die Wirksamkeit und Verträglichkeit eines häufig genutzten homöopathischen Arzneimittels mittels des «Goldstandards» für die Studiendurchführung (randomisiert, doppelblind, placebokontrolliert) überprüft werden.

Während das kombinierte primäre Zielkriterium bei einer in der Höhe unerwartet ausgeprägten Placebo-Responderrate in der Primärpopulation verfehlt wurde, zeigten die sekundären Analysen der Valid Completers einige klinische Vorteile für das Prüfpräparat. Insbesondere fanden sich hier eine Differenz der Responderraten zugunsten von Sinusitis Hevert SL und eine schnellere Linderung des wesentlichen Symptoms der Rhinorrhoe. Die primären nasalen Symptome des SNOT-20, die in der Regel besonders belastende Symptome für die Patienten darstellen (z.B. Nasenatmungsbehinderung), verbesserten sich unter Behandlung mit Sinusitis Hevert SL im Verlauf deutlicher. Die Verbesserung bei Einzelsymptomen wirkte sich auch auf eine Verbesserung der Lebensqualität aus.

Für die Interpretation zu beachten sind die trotz Randomisierung aufgetretenen Baseline-Unterschiede zwischen den Gruppen. So litten mehr Patienten der Sinusitis Hevert SL-Gruppe an Hypothyreose als in der Placebo-Gruppe. Weiter hatten Patienten der Sinusitis Hevert SL-Gruppe zum Studienbeginn eine stärker ausgeprägte Rhinorrhoe und litten etwas häufiger an einer rezidivierenden ARS. Dies könnte sowohl die Therapie-Response als auch die Remission beeinflusst haben, in diesem Falle aber zu Ungunsten des Verums.

Der in der Studie durchgeführte biphasische Dosierungsverlauf mit unterschiedlichen Arzneimittelgaben (höhere Dosierung in der 1., niedrigere Dosierung in der 2. Behandlungswoche) entspricht der zugelassenen Dosierungsempfehlung. Diese hat sich durch jahrzehntelange Erfahrung in der therapeutischen Anwendung als sinnvoll erwiesen. Aus einem Survey des Herstellers mit 499 teilnehmenden Therapeuten (Ärzte und Heilpraktiker) ist bekannt, dass bei ARS zum Teil noch deutlich höhere Dosierungen angewandt werden [21]. Aus dem Survey geht ebenfalls hervor, dass die Zufriedenheit der Therapeuten mit dem Behandlungserfolg bei ihren Patienten von der Höhe der Dosierung abhängt. Therapeuten, die eine Tagesdosierung von 16 Tabletten, z.B. durch halbstündliche bis stündliche Einnahme von 1-2 Tabletten oder mehr verordnen, bewerteten den Therapieerfolg im Mittel mit der Schulnote 1,3. Bei Dosierungen bis 8 Tabletten pro Tag wurde der Therapieerfolg mit der Schulnote 1,7 etwas weniger gut beurteilt [16]. Dies kann ein Hinweis darauf sein, dass die Wirksamkeit mit steigender Dosierung stärker ist.

Einige Limitationen der Studie sind zu beachten. Die mit über $80 \%$ sehr hohe Responderrate im MRSS $_{\text {pat }}$ weist auf eine sehr hohe unspezifische Response bzw. Placebo-Ansprechrate und/oder häufige spontane Symptomlinderung. Für diese hohe unspezifische Responderrate war die statistische Power der Studie letztlich nicht ausreichend. Andererseits ist die geringe Remissionsrate in beiden Gruppen (Sinusitis Hevert SL 31,4\% bzw. Placebo 37,4\%) vor allem auch ein Hinweis darauf, dass das Erreichen der Remission mit dem kompletten Abklingen aller 5 Rhinosinusitis-Symptome sehr streng definiert und damit als Kriterium der Therapie-Response nicht angemessen war. Daher wären für zukünftige Studien eine Population mit stärkerer Krankheitsaktivität und/oder eine höhere Fallzahl zu erwägen. Der Vorteil für das Verum in der Valid Completers-Analyse kann andererseits auch als Hinweis darauf gelten, dass sich die volle Arzneimittelwirkung nur bei zuverlässiger und auch nachgehaltener Einnahme der empfohlenen Dosis zeigt.

Weiter kann nicht abgeschätzt werden, inwiefern die partizipierenden und in Komplementärmedizin erfahrenen Therapeuten für die ausgeprägten unspezifischen placeboartigen Effekte verantwortlich waren. Vor diesem Hintergrund wäre es sinnvoll, das Verum Sinusitis Hevert SL auch in einer Studie bei ausschließlich konventionell tätigen Ärzten hinsichtlich seiner spezifischen Wirksamkeit zu überprüfen und eventuelle unspezifische Setting- und Zuwendungseffekte zu reduzieren.

Insgesamt gibt es nur wenige Studien und keine mit homöopathischen Fixkombinationen, mit denen die Ergebnisse verglichen werden können. Eine phytotherapeutische Studie mit Tabletten aus Trockenextrakt 5 pflanzlicher Inhaltsstoffe in der Indikation akute virale Rhinosinusitis weist ebenfalls auf eine hohe Ansprechrate auf Placebo mit $>85 \%$ nach 14-tägiger Behandlung hin [22].

Insgesamt zeigte sich in dieser Studie in beiden Gruppen ein vergleichbar hoher Therapieeffekt in der ITT-Population, mit Vorteilen für Sinusitis Hevert SL in einigen klinisch relevanten Symptombereichen und der Gesamt-Response in der Population der Valid Completers.

\section{Fazit}

Die vorliegende Studie zeigt, dass Sinusitis Hevert SL eine sehr gut verträgliche Therapieoption mit Wirkungen auf die Abheilungszeit und ausgewählte Symptome ist, während die GesamtResponderrate und die komplette Remission bei hoher PlaceboResponse nicht signifikant beeinflusst werden. Besonders belastende Symptome des SNOT-20 und die Lebensqualität verbessern sich unter der Behandlung mit Sinusitis Hevert SL deutlicher.

\section{Registrierung}

ClinicalTrials.gov (Identifier: NCT02296814)

EudraCT Nr. 2014-000907-29

Deutsches Register Klinischer Studien (DRKS-ID: DRKS00006877)

\section{Disclosure Statement}

A. Michalsen erhielt ein Honorar für die Arbeit am Studienkonzept und -protokoll sowie für die wissenschaftliche Beratung. J. Palm und H. Samer fungierten bei dieser Studie als Prüfärzte und haben keine weiteren Interessenkonflikte. J.-C. Wollmann und S. Tausend sind bei Hevert-Arzneimittel GmbH und Co. KG angestellt, J. Schnitker ist Direktor des Instituts für angewandte Statistik und I. Klein ist angestellt bei der AtoZ-CRO GmbH. 


\section{Literatur}

1 Rhinosinusitis. Leitlinie der Deutschen Gesellschaft für Allgemein- und Familienmedizin (DEGAM-Leitlinie Nr. 10) 2008. www.degam.de/files/Inhalte/LeitlinienInhalte/Dokumente/DEGAM-S3-Leitlinien/LL-10_ Langfassung_Rhinosinusitis-005B.pdf (letzter Abruf 24. März 2016).

2 Fokkens WJ, Lund VJ, Mullol J, et al: European position paper on rhinosinusitis and nasal polyps 2012 Rhinol Suppl 2012;23:1-298.

3 Stiärne P, Odebäck P, Ställberg B, Lundberg J, Olsson $P$ : High costs and burden of illness in acute rhinosinusitis: real-life treatment patterns and outcomes in Swedish primary care. Prim Care Respir J 2012;21: 174-179.

4 Daniller T: Management of acute bacterial rhinosinusitis. CME 2013;31:61-62.

5 Rhinosinusitis. Leitlinie der Deutschen Gesellschaft für Hals-Nasen-Ohren-Heilkunde, Kopf- und HalsChirurgie 2011.www.awmf.org/uploads/tx_szleitlinien/ 017-049l_S2k_Rhinosinusitis_2011-abgelaufen.pdf (letzter Abruf 24. März 2016).

6 Bucher HC, Tschudi P, Young J, et al: Effect of amoxicillin-clavulanate in clinically diagnosed acute rhinosinusitis: a placebo-controlled, double-blind, randomized trial in general practice. Arch Intern Med 2003; 163:1793-1798.

7 DeSutter Al, DeMeyere MJ, Christiaens TC, et al: Does amoxicillin improve outcomes in patients with purulent rhinorrhea? A pragmatic randomized double-blind controlled trial in family practice. J Fam Pract 2002;51: 317-323.
8 Merenstein D, Whittaker C, Chadwell T, Wegner B, D'Amico F: Are antibiotics beneficial for patients with sinusitis complaints? A randomized double-blind clinical trial. J Fam Pract 2005;54:144-152.

9 Stalman W, van Essen GA, van der Graaf Y, de Melker RA: The end of antibiotic treatment in adults with acute sinusitis-like complaints in general practice? A placebo-controlled double-blind randomized doxycycline trial. Br J Gen Pract 1997;47:794-799.

10 van Buchem FL, Knottnerus JA, Schrijnemaekers VI, Peeters MF: Primary-care-based randomised placebocontrolled trial of antibiotic treatment in acute maxillary sinusitis. Lancet 1997;349:683-687.

11 Lemiengre MB, van Driel ML, Merenstein D, Young J, De Sutter AI: Antibiotics for clinically diagnosed acute rhinosinusitis in adults. Cochrane Database Syst Rev 2012;(10):CD006089.

12 Rotenberg BW, Bertens KA: Use of complementary and alternative medical therapies for chronic rhinosinusitis: a Canadian perspective. J Otolaryngol Head Neck Surg 2010;39:586-593.

13 Yakirevitch A, Bedrin L, Migirov L, Wolf M, Talmi YP: Use of alternative medicine in Israeli chronic rhinosinusitis patients. J Otolaryngol Head Neck Surg 2009;38: 517-520

14 Newton JR, Santangeli L, Shakeel M, Ram B: Use of complementary and alternative medicine by patients attending a rhinology outpatient clinic. Am J Rhinol Allergy 2009;23:59-63.
15 Friese KH, Zabolotnyi DI: Homeopathy in acute rhinosinusitis: a double-blind, placebo controlled study shows the efficiency and tolerability of a homeopathic combination remedy. HNO 2007;55:271-277.

16 Fachinformation Sinusitis Hevert SL, June 2014.

17 Revicki DA, Margolis MK, Thompson CL, et al: Major symptom score utility index for patients with acute rhinosinusitis. Am J Rhinol Allergy 2011;25:99-106.

18 Piccirillo JF, Merritt MG, Richards ML: Psychometric and clinimetric validity of the 20 -item Sino-Nasal Outcome Test (SNOT-20). Otolaryngol Head Neck Surg 2002;126:41-47.

19 Baumann I, Blumenstock G, DeMaddalena H, Piccirillo JF, Plinkert PK: Quality of life in patients with chronic rhinosinusitis: validation of the Sino-Nasal Outcome Test-20 German Adapted Version. HNO 2007;55:4247.

20 Bauer P, Köhne K: Evaluation of experiments with adaptive interim analyses. Biometrics 1994;50:10291041.

21 Sinusitis-Survey 2012, unveröffentlichte Daten. Nussbaum, Hevert-Arzneimittel, 2012.

22 Jund R, Mondigler M, Steindl H, et al: Clinical efficacy of a dry extract of five herbal drugs in acute viral rhinosinusitis. Rhinology 2012;50:417-426. 